\title{
La fabrique d'une Histoire des sciences et des savoirs Entretien avec Dominique Pestre - Propos recueillis par Francesco Panese
}

Francesco Panese: Dominique Pestre, vous avez dirigé aux Éditions du Seuil une Histoire des sciences et des savoirs en trois volumes finement illustrés: «De la Renaissance aux Lumière», sous la direction de Stéphane Van Damme, «Modernité et globalisation (1770-1914)» sous celle de Kapil Raj et H. Otto Sibum, et «Le siècle des technosciences (depuis 1914)» dirigé par Christophe Bonneuil et vous-même. ${ }^{1}$ Quel était l'état de l'histoire des sciences à partir duquel vous avez voulu concevoir et développer ce grand projet?

Dominique Pestre: Ce travail est parti d'un constat historiographique et d'un phénomène massif, la transformation que les sciences studies ont induite depuis les années 1970: on s'est mis à écrire autrement l'histoire des sciences, à la regarder aussi comme pratique matérielle et sociale par exemple, à la prendre d'une manière moins idéalisée, plus anthropologique. Cet apport fait la force de cette historiographie: elle part du fait que les sciences sont prises dans le monde, qu'elles sont prises dans l'économique et le politique - et ne pas séparer ces éléments s'est avéré très productif. En revanche, et ceci aussi est surprenant, les grandes histoires des sciences sont restées relativement classiques et plutôt disciplinaires. C'est à partir de cet écart que le projet a été pensé. Lorsque les Éditions du Seuil me l'ont proposé, il était clair que nous devions profiter pleinement de la floraison extraordinaire que nous avions connue en quarante ans, et tenter de placer toutes ces micro-analyses de situations dans des visions de longue durée. Mais ceci nous mettait face à une difficulté majeure, celle de s'engager dans une histoire devenant quasi-

1 Dominique Pestre (éd.), Histoire des sciences et des savoirs, Paris, Éditions du Seuil, 2015. Vol. 1, Stéphane Van Damme (éd.), «De la Renaissance aux Lumière» [ISBN: 978-2-02107676-9]; Vol. 2, Kapil Raj et H. Otto Sibum (éds), «Modernité et globalisation (1770-1914)» [ISBN: 978-2-02-107677-6]; Vol. 3, Christophe Bonneuil et Dominique Pestre (éds), «Le siècle des technosciences (depuis 1914)» [ISBN: 978-2-02-107678-3]. 516 pages, € 38 (par volume).

Francesco Panese, Institut d'histoire de la médecine et de la santé publique (CHUV-FBM/ UNIL) et Faculté des SSP (UNIL); francesco.panese@unil.ch 
totale, dans laquelle «tout est dans tout»- un travail impossible. Il a donc fallu gérer cette tension.

FP: Vous dites dans votre introduction que vous avez voulu «prendre à bras le corps des questions souvent marginalisées dans les grandes histoires des sciences». Quelles sont ces questions?

DP: Pour répondre d'un mot et reprendre le titre d'une célèbre revue, ce sont celles qui relèvent de la science in context. Et dans les grandes histoires des sciences, nous sommes peu dans la science en contexte. Je pense que cela tient au fait que faire une histoire de la longue durée nécessite de dépasser les situations locales et de suivre un ou plusieurs fils directeur. On peut par exemple prendre le fil des paradigmes, des concepts, des disciplines; ce sont de bons fils directeurs qu'il n'y a aucune raison de rejeter, et dans notre ouvrage nous avons toujours une section qui s'appelle «Champs de sciences» qui répond, je crois, à cette exigence intellectuelle. Mais nous sommes plutôt partis du parti pris qu'il y avait d'autres questions à ne pas manquer, des questions transversales que nous devions traiter elles aussi, avec la même exigence. Par exemple, qu'il fallait rendre présentes les dimensions pratiques et corporelles de l'activité scientifique, que soient intégrées les questions économiques et de pouvoir, celles du gouvernement, du local au gouvernement à distance; mais encore celles des races et du genre, des relations entre l'Europe et le reste du monde, etc.

FP: La multiplication de ces questions ne va-t-elle va de pair avec la porosité des frontières entre les sciences et leurs contextes, les sciences et les autres savoirs?

DP: Oui. On peut dire que, aujourd'hui, nous définissons moins les sciences de l'intérieur que dans leurs liens aux autres formes de savoir et aux espaces dans lesquelles elles s'élaborent. En d'autres termes, on peut dire qu'il n'y a plus de naturalité de «la science». C'est un héritage d'auteurs comme Harry Collins et David Bloor dans les années soixante-dix, de Bruno Latour et des études féministes dans les années quatre-vingt: la science est toujours située dans ce qu'elle produit, il n'y a pas de frontières nettes séparant les sciences des autres formes de savoir, et nous ne considérons plus que les sciences se déploient sans nombre de savoir-faire. Tout en assumant le risque de l'histoire totale, nous avons dès lors tenté de dimensionner notre projet en nous demandant, pour chaque volume, quels étaient les rapports aux savoirs non académiques, quels étaient les rapports aux savoirs non-européens et aux 
autres pratiques sociales comme la production de biens ou le commerce. Mais cette interrogation ne pouvait rester générale et il a fallu y répondre en faisant des choix, des choix articulés pragmatiquement et au cas par cas.

FP: Cette histoire par cas se présente aujourd'hui comme une histoire plurielle des sciences et des savoirs. Comme avez-vous résolu la question des limites qu'il fallait forcément lui donner?

DP: Sur le plan intellectuel, le travail s'est fait en deux temps. Dans une première phase, nous avons réfléchi à la meilleure manière de quadriller ce que nous voulions dire et ce qu'il ne fallait pas oublier. Nous nous sommes par exemple demandé, pour chaque époque, quels étaient les objets scientifiques par excellence - la cellule pour le biologiste, la force pour le physicien, etc. Nous nous sommes posé la même question pour les types d'instruments qui marquent symboliquement chaque période - les instruments auto-enregistreurs du XIX ${ }^{\mathrm{e}}$ siècle par exemple. Le résultat était un nombre de paramètres très grand (trop grand pour être gardé) mais, dans un premier temps, il s'agissait de ne pas tuer la floraison. Encore une fois, plutôt que de penser par théorie, nous avons adopté la pensée par sujets, types de pratiques et lieux. Dans un second temps, nous avons divisé les volumes en vingt à vingt-deux chapitres avec la contrainte que chacun d'eux raconte son histoire. Mais au niveau de chaque volume, nous devions nous assurer que l'ensemble offrait au lecteur une vision globale et pertinente de l'époque, un sentiment du temps. Dans un dernier temps, ceci s'est traduit par des demandes spécifiques à des auteurs à qui nous avons dit: «Votre contribution s'inscrit autour de cette question, mais vous devez vous sentir libre de la prendre comme vous le souhaitez, de nous donner votre sentiment.» C'était une manière de leur suggérer de travailler sur le mode de l'essai, de dire ce qui leur semblait au cœur de la question posée.

FP: Pouvez-vous illustrer ce point en expliquant concrètement comment vous avez procédé dans ces choix?

DP: Prenons un exemple concret, celui des mathématiques et de la manière de les considérer. Pour nous, cela n'avait pas de sens de faire une histoire des mathématiques pour chaque siècle; cela aurait obligé les auteurs à rester superficiels et débouché sur une histoire plutôt disciplinaire, deux choses que nous voulions éviter. Nous avons donc fait le choix de thèmes spécifiques pour chaque époque, l'ensemble devant toutefois faire émerger des approches complémentaires. Dans le premier volume par exemple, nous avons privilé- 
gié les «mathématiques pratiques». D'autres choix auraient bien sûr été possibles, mais nous avons décidé que celui-ci était le plus intéressant, puisque c'est à travers cette notion que les mathématiciens se définissaient le plus souvent, et qu'il permettait de montrer les liens étroits existant entre savoirs mathématiques, astronomie, architecture, cartographie, maîtrise du territoire et affaires militaires (fortification et artillerie). Pour l'époque contemporaine, nous avons procédé de même et avons considéré que la notion de «modélisation» était une bonne entrée. Le «modèle» naît en mathématique dans les années 1920, notamment avec Volterra en Italie, et il permet de rendre compte d'une variété de situations, de la simulation des bombes $\mathrm{H}$ en 1950 au changement climatique aujourd'hui. Cette manière de procéder a l'avantage de faire surgir des thèmes généraux - par exemple «modèle et simulation»- et de les actualiser dans des cas spécifiques. Et une fois ces premiers choix effectués, nous revenions sur ce qui avait disparu des radars et qu'il fallait reprendre. Ce qui nous a conduit à retenir, pour le deuxième volume par exemple, le thème des «mathématiques pures» (pures au sens où les acteurs l'entendent alors) - ce qui est au cœur du XIX ${ }^{\mathrm{e}}$ siècle. Ce choix, à son tour, ouvrait sur le débat avec les «mathématiques romantiques», qui nous conduisait à un autre univers. Prenons un autre exemple, celui des «sciences de la terre»: celles-ci sont traitées dans le premier volume sous l'angle de la cartographie - en lien à ce qui importe alors, les grandes découvertes et les mathématiques pratiques; on les retrouve dans le second sous l'angle de la physique et des grandes campagnes internationales de mesure (du magnétisme terrestre par exemple), une grande nouveauté du second XIX ${ }^{\mathrm{e}}$ siècle; et dans le troisième volume sous l'angle de la fabrication, de la calibration et du traitement des données, terrestres et spatiales, liées à la surveillance des changements climatiques. Ce faisant, nous avons privilégié l'objet Terre - et non des disciplines spécifiques telle que la géologie -, et montré les pratiques de sciences diverses qui ont été mobilisées au fil du temps. Autre exemple encore, nous avions prévu une entrée «institutions globales» en nous demandant quelles étaient celles qui, à chaque moment, ont compté dans l'histoire des sciences et des savoirs. Pour l'époque moderne, nous avions deux grandes options, les ordres religieux d'une part, les grandes compagnies de commerce anglaises ou néerlandaises de l'autre, deux types d'institutions qui collectent alors des savoirs à l'échelle globale et les traitent au «centre», en Europe; pour le $\mathrm{XX}^{\mathrm{e}}$ siècle, nous hésitions entre la Banque mondiale, qui se définit comme la «banque des savoirs», et les grands programmes des NationsUnies, ce qui nous permettait d'intégrer la Société des Nations. Mais il reste encore une exigence puisqu'il convient d'équilibrer les volumes eux-mêmes, de leur donner de la cohérence et de vérifier que rien d'essentiel n'est oublié 
- ce qui conduit à d'autres ajustements et, finalement, aux volumes que vous avez sous les yeux.

FP: Pour mener à bien ce travail, pourrions-nous dire que vous vous êtes en quelque sorte nourris des travaux d'une communauté d'historiens pour cartographier à la fois des domaines et des thèmes spécifiques, et que cela vous a permis de retourner vers eux pour leur passer commande?

DP: C'est bien cela. Encore une fois, il faut bien prendre la mesure de l'extrême richesse, de la profusion sidérante des travaux réalisés depuis trois à quatre décennies. Nous sommes bien sûr partis d'elle en nous demandant comment mettre un peu d'ordre dans cette profusion. Nous l'avons fait en établissant des tableaux synoptiques formés de dizaines de lignes (chacune sur un thème, une préoccupation, une idée) et dont les colonnes étaient les différents volumes que nous avions retenus. Il a bien sûr fallu ensuite resserrer et, au dernier moment, évidemment, choisir les personnes en qui nous avions confiance et à qui demander une contribution. Et forcément, chemin faisant, certaines des lignes de nos grands tableaux ont disparu et le projet a été refaçonné par ce que les auteurs nous ont concrètement remis; car, comme je l'ai dit, nous ne souhaitions pas trop les contraindre pour leur permettre de raconter leur histoire, avec leurs exemples, leur épaisseur anthropologique, leur style - et ce qu'ils voulaient vraiment nous dire. Ceci étant, j'espère qu'on peut encore repérer, à la lecture des trois volumes, ces formes de cohérence - même si l'on est face, en même temps, à soixante-dix histoires différentes. Pour finir, nous avons défini un dernier niveau de lecture formé d'introductions longues rédigées par les directeurs de chaque volume; par une conclusion générale, que je propose en fin d'ouvrage; et par la mise en évidence d'un plan assez stable de volume à volume. En règle générale, chaque volume considère d'abord la place des sciences dans l'ordre social, culturel et économique. Puis les champs de sciences qui marquent la période. Puis les phénomènes de mondialisation et la production «des autres». Enfin le rôle des sciences dans le gouvernement du monde.

FP: C'est en effet l'impression que donne l'ensemble dont les sections commencent par des essais qui embrassent des thématiques assez larges et qui sont suivis par d'autres qui se resserrent sur des objets plus spécifiques.

DP: C'est vrai. Nous avions le projet d'ouvrir les sections sur des cadrages généraux pour entrer ensuite dans des histoires plus particulières. Nous avions par exemple choisi d'ouvrir chaque volume par un chapitre intitulé «Figures 
de savants» ou «Figures de scientifiques»: dans le premier volume, c'est ce que fait J.B. Shank dans son excellente contribution; dans le troisième, c'est celle de Steve Shapin, très originale dans sa manière de traiter la question sous un angle quantitatif. Mais le lecteur notera qu'un tel chapitre est absent du deuxième volume - simplement parce que l'auteur pressenti n'a pas été en mesure de le rendre. Ce sont des choses qui arrivent. Mais nous avons eu la chance de recevoir la contribution de John Pickstone avant qu'il ne nous quitte ${ }^{2}$; et son essai «Révolutions analytiques et les synthèses du modernisme» joue très bien ce rôle de mise en perspective générale pour le volume 2 .

FP: Revenons sur la forme des contributions qui composent ces trois volumes. Vous avez mentionné à plusieurs reprises le genre de l'«essai». Qu'est-ce qui le caractérise le mieux à vos yeux?

DP: Je crois tout d'abord que toutes les contributions ne correspondent pas forcément à ce genre, ou en tous cas pas de la même manière. Je n'ai pas forcément de définition précise de ce qu'est un «essai». Je répondrai simplement que nous avons suggéré aux auteurs de prendre la liberté de ton qu'ils souhaitaient, qu'ils n'avaient pas à se soumettre à la norme qui tend à prévaloir dans les articles de revue. Dans nos domaines, surtout dans les grandes revues, nous sommes aujourd'hui très cadrés, à l'instar des articles des sciences dites «dures» dont le modèle s'étend. Par exemple, bien que nous demandions aux auteurs de couvrir des périodes historiques assez longues, nous leur avons suggéré de ne pas commencer par les traditionnelles revues de la littérature, trop encyclopédiques, pour ne pas contraindre indûment les interprétations historiographiques que nous espérions. Nous leur avons demandé de trouver eux-mêmes le fil conducteur, de raconter leur histoire de façon concrète et située, et de ne pas hésiter à faire part au lecteur de leur sentiment profond, de ce qu'il convenait de comprendre - de partager avec nous des thèses fortes, même dans un format court. En d'autres termes, et sans vouloir être grandiloquent, nous leur avons donné la possibilité de sortir du cadre strict du «bien faire scientifique» et d'ouvrir largement leurs propositions.

FP: Ce que vous dites renvoie à un sentiment très fort que nous avons en lisant ces trois volumes: de grandes compétences réunies en un projet pédagogique. Cette histoire a, me semble-t-il, aussi les allures d'un nouveau type de manuel d'histoire des sciences.

2 L'historien des sciences britannique John Pickstone est malheureusement décédé le 12 février 2014. 
DP: Je suis d'accord avec vous. Et je crois que cela est lié à l'idée de développer des pensées fortes et originales dans chaque chapitre, mais couplées à une complémentarité organisée des contributions. Prenons le troisième volume que je connais bien pour l'avoir personnellement dirigé avec mon ami Christophe Bonneuil et regardons comment se construit la section sur les champs de siences. La contribution de Jacques Revel ouvre le bal et porte sur «L'avènement des sciences sociales» - une invention majeure de la fin du $\mathrm{XIX}^{\mathrm{e}}$ siècle. Son choix a été de nous proposer une vue panoramique des traditions complexes qui font ce champ, et des différences culturelles qui persistent entre les Etats-Unis, la France, l'Allemagne etc. Mais cet article est immédiatement suivi par la contribution de Sarah Franklin, «Foucault et les transformations du biopouvoir», dans laquelle elle prend les sciences sociales à une toute autre échelle, avec une toute autre préoccupation: elle avance une généalogie neuve du concept foucaldien de biopolitique (en le mettant notamment en lien avec la question de la parenté chez Claude Lévi-Strauss) et en discute les effets dans le champ devenu très important de l'anthropologie de la biomédecine. Emerge alors un autre univers, une autre dimension des sciences sociales. Poursuivant sa lecture, le lecteur passe à un article consacré à la montée en puissance et aux transformations radicales de l'économiediscipline, par Timothy Shenk et Timothy Mitchell. Ce chapitre retrace comment ce champ s'autonomise des autres sciences sociales, entre en partie en opposition à elles, et devient un outil majeur de gouvernement et de transformation des idéologies sociales. Mais les sciences sociales sont encore présentes dans le chapitre suivant, qui porte sur «Les savoirs de la diversité humaine»; il implique cette fois des sciences dures mais se doit de répondre aux désastres des sciences de la race qui dominent la première partie du $\mathrm{XX}^{\mathrm{e}}$ siècle. Et cela continue ainsi, essais après essais, sur les approches écologiques, des ONG aux savants, sur la suprématie du gène en biologie, etc.

FP: Le tissage des thématiques et des perspectives que vous décrivez pose aussi la question des relations entre l'histoire et les science studies que vous avez mentionnées. Quelles sont à vos yeux ces relations aujourd'hui?

DP: Vous adressez cette question à un historien, un historien qui s'occupe des sciences mais qui est avant tout un historien au sens généraliste du terme. Pour moi, histoire et science studies relèvent de genres assez différents, mais il y a continuité et stimulation réciproque entre les deux types de travaux; les science studies constituent un champ d'investigation capital, plus théorique, plus libre dans la définition de ses objets, où la question des sciences a été radicalement reposée, où ce qui lie ces dernières aux autres pratiques sociales 
et aux autres formes de savoir a été finement commenté - et qui a proposé d'autres outils, dont les analyses de controverses «symétriques», et des mises au point théoriques de premier ordre, dont l'absence de solution de continuité entre les sciences et les savoir-faire, par exemple. Comme historien, je crois que c'est le meilleur angle d'approche, celui qui permet l'écriture des histoires les plus intéressantes et contextualisées. Mais, bien sûr, on peut aussi écrire des histoires conceptuelles passionnantes, et on doit le faire, à la manière de Georges Canguilhem, et sans avoir alors à se soucier beaucoup des sciences studies. En ce qui me concerne, j'ai appris beaucoup des science studies - parce qu'elles m'ont ouvert à une histoire des sciences qui puisse être pratique et matérielle, politique et économique, sociale et culturelle. Et c'est l'ensemble de ces aspects qui me semblent heuristiquement intéressants dans les science studies ${ }^{3}$.

FP: N'est-ce pas en ce sens que va votre intérêt pour ce que vous appelez les «formes de vie et d'expériences»?

DP: Je n'ai pas de théorie générale sur cette question. Je dirais que j'emploie banalement l'expression de «forme de vie» dans le sens qu'elle a prise dès les années quatre-vingt, lorsque Ludwig Wittgenstein est redevenu fortement présent, au Royaume-Uni notamment. Je crois que dans le genre d'entreprise qui est la nôtre avec cette Histoire, l'objectif n'est pas d'abord de simplifier les choses, mais de commencer par montrer la variété des formes d'être, puis de mettre en évidence que cette variété a des règles que l'on peut caractériser sur des périodes temporelles. Si l'on se pose par exemple la question des sciences physico-chimiques au $\mathrm{XX}^{\mathrm{e}}$ siècle, on pense forcément à la guerre, à l'économie, au rôle de l'État, à la mystique du progrès, à l'importance de la promesse, au rôle des media et des images, aux pollutions et désastres écologiques. Et vous voyez que cela ordonne les premiers chapitres du troisième volume, qui sont autant de manières d'aborder ces questions des sciences «en société». Je crois que la bonne manière en histoire, comme dans l'ensemble des sciences sociales, est de varier systématiquement les regards et angles d'approches, les échelles et cadrages, les questions et manières de découper les objets - car ce que nous avons à comprendre est trop vaste et complexe pour être saisi en un seul mouvement. Il faut systématiquement «tourner autour de nos objets», de ce que l'expression «sciences et savoirs» implique dans l'ensemble de l'ordre social, de la saisir comme ensemble de relations.

3 Voir: Dominique Pestre, Introduction aux Science Studies, Paris, La Découverte, coll. Repères, 2006. 
C'est ce que nous avons tenté de faire de manière presque obsessionnelle avec, par exemple, les relations de «genre», de «race» et la «globalisation». Prenons la «race» par exemple. Dans le premier volume, elle est déclinée par Jean-Frédéric Schaub et Silvia Sebastiani sous l'aspect de l'émergence des «Savoirs sur l'autre» et de la manière dont ils se réordonnent au XVIII siècle; dans le second volume, nous la prenons deux fois, sous l'angle de la mondialisation et des représentations cartographiques des races d'une part, sous l'angle plus biologique et administratif de l'hérédité et de l'eugénisme de l'autre. Dans le troisième volume, finalement c'est sous l'angle scientifique que nous reprenons la question - via la manière dont la question est effacée sans être pleinement résolue à travers le concept de «diversité humaine».

FP: En revenant, si vous le voulez bien, à l'ensemble de cet immense ouvrage et en considérant son titre, pourriez-vous expliciter les distinctions que vousmême et les auteurs faites entre «sciences» et «savoirs»?

DP: Dans le cadre des héritages des science studies, je dirais que la première chose que nous avons apprise est qu'il est heuristiquement plus riche de ne pas postuler une différence d'essence entre «la science» et «le reste». Si une telle différence était non problématique, il n'y aurait pas de polémiques (entre disciplines et traditions scientifiques par exemple) et nous n'aurions pas à écrire une histoire des sciences et des savoirs. Non qu'il n'y ait pas de différences entre physique des particules et savoirs artisans - affirmer le contraire serait ridicule. Mais il y a peut-être autant de différences entre cette physique et la théorie de l'évolution ou l'histoire-discipline qu'avec l'épidémiologie populaire. Nous postulons plutôt des ordres de continuité entre diverses formes de savoir, et entre savoirs, faire, et savoir-faire, postulat de travail bien illustré par Otto Sibum lorsqu'il analyse «les sciences et les savoirs traditionnels» au XIX ${ }^{\mathrm{e}}$ siècle en Allemagne. Son point est de montrer la place incontournable des métiers et de la production dans l'émergence de la reine des sciences elle-même, la physique de précision; et il montre le rôle clé de la circulation des manières de faire, des hommes et des instruments pour que la nouvelle physique puisse se constituer. Pour nous, au point de départ de l'enquête, la reconnaissance que «ceci est la science» n'est donc pas prise comme une évidence mais comme une affirmation qu'il convient d'analyser. Il n'y a pas d'essence du «scientifique», et c'est dans l'action et en situation que l'évidence s'énonce et, éventuellement, se solidifie. Notre travail consiste à suivre et cartographier les manières dont les acteurs eux-mêmes établissent ces distinctions. Pensons à l'«Affaire Mesmer», à la fin du XVIII siècle, durant laquelle l'Académie affirme la distinction entre le 
«charlatanisme» de la pratique du médecin autrichien et la «science» des experts chargés de la mettre à l'épreuve. La suggestion des science studies est que nous, historiens, devons d'abord comprendre comment l'Académie fait le partage et comment ceux qui s'opposent à elle le contestent; elle est de «suivre les acteurs en action», comme dit Bruno Latour, sans commencer par intervenir dans le débat lui-même et distribuer bons et mauvais points. Pour revenir à votre question, il s'agit, par notre titre, de dire au lecteur que nous lui proposons une histoire des formes de savoirs rencontrés à l'échelle planétaire depuis cinq siècles, de leurs modes d'interaction et de traduction, des recompositions qui émergent de ces rencontres - pas une histoire simple de «la science» sortant toute armée et sans perte de grands esprits. Et si le mot «sciences» se trouve dans le titre, c'est que celles-ci, dans la diversité de leurs pratiques, sont une forme particulièrement puissante de savoir en termes intellectuels autant que pratiques, économiques autant que politiques ou militaires. C'est aussi, point capital pour nous, que beaucoup des sciences que nous pratiquons se sont redéfinies lors des rencontres qu'ouvrent les grandes découvertes; qu'elles se sont recomposées au travers des échanges comme autour du transfert des plantes et des savoir-faire, et que les inputs viennent de toutes parts - et non d'abord d'un centre européen qui aurait fait «la révolution scientifique» et inventé «les sciences» d'un trait de génie et à lui tout seul. En cela nous proposons, après d'autres, une autre histoire du monde.

FP: En me demandant à quel autre projet pourrait être comparé le vôtre, j’ai pensé à celui mené par Christian Jacob sous le titre «Les lieux de savoir», ces deux imposants volumes collectifs ${ }^{4}$ qui abordent, dans la diversité des temps et des cultures, les manières dont on a produit, validé, sauvegardé et transmis des savoirs, au sens très large du termes. Partagez-vous cette possible comparaison?

DP: Oui, manifestement. Christian Jacob et moi enseignons au même endroit, au Centre Alexandre Koyré (à l'Ecole des Hautes Etudes en Sciences Sociales) et nous partageons en grande partie les mêmes postulats de départ. Je dirais que Les Lieux de savoir privilégie avant tout, comme son titre l'indique, les lieux, les espaces. Cette perspective m'est également chère et j'avais dirigé en son temps un numéro spécial de La Recherche sur le thème des «lieux de science» ( $\mathrm{n}^{\circ}$ 300, juillet-août 1997). La vingtaine de contributions qui y étaient rassemblées faisaient voyager le lecteur dans des lieux que nous

4 Christian Jacob (éd.), Les lieux de savoir. Vol. 1: «Espaces et Communautés», Paris, Albin Michel, 2007, 1282 p. [ISBN 9782226179043]; vol. 2 «Les Mains de l'intellect», Paris, Albin Michel, 2011, 992 p. [ISBN 9782226187291$].$ 
retrouvons en partie dans notre Histoire des sciences et des savoirs: le champ de bataille à la Renaissance, l'Académie à Paris, les compagnies de commerce, la Compagnie de Jésus, l'Institut Pasteur, l'Observatoire de Pulkova, les laboratoires de la compagnie Bell, la Rand Corporation, la salle de rédaction de la revue Nature, etc. - autant de lieux de fabrication de science. Je dirais qu'à partir de cette perspective partagée, ce sont toutefois moins les lieux qui ont structuré notre travail - même s'ils sont omniprésents - que la question de ce qui fait une situation - soit une question plus classiquement historienne. Notre objet principal est de comprendre des configurations, ce qui définit au temps $t$ les lieux et modes de production et de circulation des sciences et savoirs, la variété de leurs porteurs, les conflits de légitimité qui les opposent ou les allient, l'articulation de ces savoirs à l'espace public, les ensembles conceptuels, les formes de vie et les imaginaires qui les parcourent. Par exemple, dans le premier volume, la question qui nous guide est: qu'est-ce qui fait, quelle est la nature de cet «ancien régime des sciences et savoirs» qui court entre $\mathrm{XVI}^{\mathrm{e}}$ et $\mathrm{XVIII}{ }^{\mathrm{e}}$ siècles? Par exemple encore, la contribution de Norton Wise «Comment marche le monde», dans le second volume, analyse les «trois approches» qui, comme il le montre, caractérisent la variété du travail des physiciens de l'époque. De même, Christophe Bonneuil, dans «Le siècle du gène» (volume 3), déploie son analyse à partir de l'obsession réductionniste qui parcourt les sciences du vivant au $\mathrm{XX}^{\mathrm{e}}$ siècle; et il montre non seulement comment elle devient le cœur organisateur de la biologie, mais aussi comment cette réduction opère dans «trois formes d'économies politiques différentes». D'autres contributions ont pour pivot les instruments, telle celle de Paul N. Edwards «Gouverner le système Terre» (volume 3), qui décrit la mise en place des systèmes de monitoring de la planète. D'autres encore articulent des «espaces» et des «formes sociales», comme celle de Natalia Muchnik «Dynamiques transnationales et circulations diasporiques des savoirs» (volume 2). En résumé, je dirais que toutes ces contributions n'envisagent pas que des lieux, mais que les lieux sont toujours là car ils ne peuvent jamais être ignorés. Et certaines contributions auraient pu trouver leur place dans le travail de Christian Jacob lui-même - par exemple la contribution de Nicholas Dew «Un colbertisme scientifique» (volume 1), centrée sur le bureau de Colbert et qui montre comment un lieu de fabrication du savoir - ici le savoir de l'administration - est toujours articulé sur un «projet», en l'occurrence le projet d'une souveraineté politique.

FP: Ce que vous dites montre bien votre volonté de proposer des histoires contextuelles qui convergent vers une histoire des situations dans lesquelles les savoirs sont à la fois les symptômes, les emblèmes et les vecteurs de façon- 
nage de ces situations. Et dans ces situations, me semble-t-il, le politique occupe une place importante, sinon cardinale. Vous expliquez dans l'introduction générale l'importance à vos yeux de rendre compte des relations entre science et politique, sous l'angle en particulier, dites-vous, de ce qui «transforme le savoir pertinent ou socialement utile» (vol. 1, p. 12). En considérant l'ensemble de ce projet, pouvez-vous expliciter ces relations entre science et politique, les relations entre sciences, savoirs et «vie sociale»?

DP: Il ne fait pas doute que la dimension politique - entendue au sens général des relations de pouvoir et de l'organisation de l'ordre social - ressort massivement de l'ensemble de l'ouvrage; c'est même, je crois que vous avez raison, une tonalité dominante des trois volumes. J'en ai été frappé moimême à la lecture finale de l'ouvrage et j'ai souhaité y revenir dans les huit thèses que je développe dans mes conclusions. Premier point, les globalisations constituent une ligne de force de nos volumes - ce qui semble bien banal si l'on considère l'ensemble des savoirs et qu'on ne se limite pas à ce qui est né en Europe et qui a été repris et adapté dans d'autres contextes -, pensons à la physique newtonienne par exemple. Pour la plupart des savoirs, en effet, ceux qui relèvent du droit, des sciences naturelles, de la géographie, de l'anthropologie, des techniques, des savoirs de la navigation, de la médecine, de la pharmacopée et d'autres, l'hypothèse d'une fabrication en Europe qui se serait ensuite diffusée est fausse, ou du moins très incomplète. Dans les contextes reconfigurés par les grandes découvertes par exemple, c'est bien localement que les choses émergent, c'est dans la rencontre que beaucoup de choses naissent. Mais il convient aussi de rendre compte du fait que cela se passe dans un ordre souvent asymétrique, un ordre politique, un rapport de forces donné. En d'autres termes, les savoirs sont bien radicalement coproduits, mais dans un champ de force qui n'est pas isotrope. Pour reprendre la notion de Bruno Latour, il est certain qu'existent des centres de calcul à Calcutta ou à Mexico, mais les grands centres de calculs sont à Londres, à Amsterdam, à Paris - puis à Berlin et aux Etats-Unis (mais peut-être aujourd'hui aussi en Chine). Le deuxième point qui m'a frappé en lisant l'ensemble des trois volumes avant d'écrire ma conclusion a été de constater que le commerce était le véhicule premier de la circulation et de la recomposition des savoirs. En effet, si l'on comprend que les savoirs sont toujours inscrits dans des objets concrets - des livres imprimés et des manuscrits, des plantes et des animaux, des technologies et des instruments - force est de constater que leurs grands médiateurs sont les commerçants. En exagérant un peu, on peut dire qu'Amsterdam en 1700 est le centre du monde des savoirs globaux parce que c'est le centre du commerce. La circulation des savoirs apparaît en 
effet très liée aux opérations de commodification (des plantes médicinales, des objets d'art, des ouvrages), et cette commodification repose sur des pratiques de savoir. Par exemple, la commodification des plantes «exotiques» qui vont peupler les jardins occidentaux repose sur l'étude de leurs propriétés, sur les classements qu'on opère, sur l'attractivité qu'on leur attribue. Il en va de même pour les manuscrits qui arrivent du sous-continent indien par exemple: pour qu'ils acquièrent de la valeur, il convient qu'ils soient décrits, documentés, traduits, annotés, édités. Nous savions déjà le rôle central joué par le commerce, mais en relisant ces trois volumes, la transversalité de cet aspect se révèle sidérante. Troisième élément, le rôle de l'État. Dans la diversité de ses formes, il est l'un des espaces de production les plus importants qui soient, aujourd'hui comme en 1500. Nous le savions aussi grâce aux travaux de David Edgerton, qui a contribué au troisième volume, mais à nouveau, en relisant l'ensemble, du bureau de Colbert aux centres d'innovation états-uniens d'aujourd'hui en passant par les administrations et les données qu'elles collectent pour gouverner le monde, ce rôle s'est révélé lui aussi cardinal dans l'élaboration des sciences, dans ce qu'elles étudient et tendent à oublier - et ceci en Europe comme en Asie ou dans les Amériques. Le gouvernement par les sciences et la connaissance des populations, des lieux, et des formes de vie sont autant de conditions nécessaires à la conduite des conduites, si chère à Michel Foucault. Quatrième élément, les mondes de la production. On connaissait bien sûr aussi le rôle des mondes industriels dans l'usage et la production des sciences et des savoirs, mais la production et la circulation des objets matériels manufacturés sont apparues elles aussi comme décisives dans l'évolution des sciences et savoirs.

Deux dernières conclusions ont été plus généreuses en retour de ce que j'escomptais lorsque nous avons conçu les volumes et distribué les textes. L'une concerne les relations entre les sciences et l'espace public: A la remise des textes, nous nous sommes rendu compte de ce que nous savions mais sans bien le savoir: l'omniprésence du passage par l'espace public; non seulement par le politique qui l'utilise - des entrées royales aux médias contemporains; non seulement par le commerce et le monde industriel; mais aussi et spécifiquement par les sciences qui s'y donnent toujours à voir. On retrouve là la thèse du premier Latour, dans son livre sur l'itinéraire de Louis Pasteur ${ }^{5}$, et qui montre combien l'espace public peut légitimer les savoirs et rendre leurs auteurs incontournables (Pouilly-le-Fort!). Et la surprise a été de voir émerger une véritable chronologie des espaces publics pertinents pour le déploie-

5 Bruno Latour, Pasteur: Guerre et paix des microbes, suivi de Irréductions, Paris, La Découverte, coll. «Sciences humaines et sociales», 2001 [1̀re éd. 1984]. 
ment des sciences. Pensons ici au XIX ${ }^{\mathrm{e}}$ siècle et à la manière dont les expositions internationales mettent en scène les sciences via les instruments et les objets manufacturés. Nous avions enfin incité nos auteurs à être attentifs à la catégorie de «nature», dans son sens le plus ouvert. Et là aussi la surprise a été de constater à quel point, depuis l'époque moderne, non seulement des scientifiques parlent de la nature et de nos environnements, mais qu'ils parlent très tôt et de manière récurrente de sa destruction par la civilisation, bien avant l'engouement actuel pour cette question. L'article de Fabien Locher dans le deuxième volume - «Changement climatique et colonisation. Amériques et Océan Indien» - est de ce point de vue extrêmement éclairant en ce qu'il montre comment, très tôt, la question de la préservation des forêts a été liée au souci du climat, de peur de rendre le monde désertique, ou encore glacé. Ainsi, dès le XVIII ${ }^{\text {e }}$ siècle, cette obsession est apparue au cœur des activités de nombres de savants et d'administrations.

FP: Vous mettez ici en lumière un point effectivement très saillant de ce grand projet: un très grand nombre de contributions permettent au lecteur de redonner de la durée à bon nombre de questions qui configurent encore et aussi notre actualité. Ceci me conduit à vous poser la question de la périodisation, de la chronologie que vous avez adoptée et sur laquelle vous revenez dans votre conclusion générale.

DP: La question est bien sûr importante et conceptuellement intéressante. Nous savons que toute périodisation a sa part d'arbitraire. Mais on peut relever aussi que, dans un tel projet, l'éditeur joue un rôle important - par exemple en fixant le nombre de volumes. Trois volumes semblaient un optimum aux Éditions du Seuil pour pareil sujet; et elles semblent ne pas s'être trompées puisque la première édition a été intégralement vendue. Mais mon idée initiale était un projet en quatre volumes, selon une autre chronologie que je propose dans ma conclusion. J'y insiste sur l'intérêt intellectuel qu'il y aurait eu à découper les deux derniers siècles et demi en trois phases et non deux, l'une allant des années 1770 aux années 1870, première forme de la modernité scientifique et politique; la suivante qui irait de ce cap de la seconde révolution industrielle aux années 1960, et qui dirait la seconde forme de la modernité, celle de la techno-industrie triomphante; et les 50 dernières années, depuis le tournant des années 1960-1970, et qui pourraient être définies possiblement par quatre mots: biotechnologie, numérique, environnement, libéralisation économique. Cette chronologie aurait eu l'avantage de la cohérence vis-à-vis de nombre d'éléments ayant trait au politique et à l'histoire de l'Etat, aux modes de la production et de la régulation, aux formes 
d'existence de la «société civile» et de la «société politique» - chaque élément s'articulant sur des sciences et des formes de savoir particulières. Car les décennies qui terminent le XVIII ${ }^{\mathrm{e}}$ siècle, comme celles de la fin du $\mathrm{XIX}^{\mathrm{e}}$ siècle et le basculement des années 1960-1970 manifestent un grand renouvellement des pratiques de sciences et de leurs liens au monde. Bref, je crois que nous aurions proposé de meilleures cohérences encore en quatre volumes. Mais nous avons tenté de le faire en trois, en espérant y avoir réussi avec les contraintes, mais aussi les grandes libertés dont a bénéficié ce projet. 